\title{
BMJ Open Prevalence of peritonitis and mortality in patients treated with continuous ambulatory peritoneal dialysis (CAPD) in Africa: a protocol for a systematic review and meta-analysis
}

\author{
Mothusi Walter Moloi, ${ }^{1,2,3}$ Shepherd Kajawo, ${ }^{1,3,4}$ Jean Jacques Noubiap, ${ }^{5}$ \\ Ikechukwu O Mbah, ${ }^{6}$ Udeme Ekrikpo, ${ }^{1,3,7}$ Andre Pascal Kengne, ${ }^{5,8}$ Aminu K Bello, ${ }^{9}$ \\ Ikechi G Okpechi ${ }^{1,3}$
}

To cite: Moloi MW, Kajawo S, Noubiap JJ, et al. Prevalence of peritonitis and mortality in patients treated with continuous ambulatory peritoneal dialysis (CAPD) in Africa: a protocol for a systematic review and meta-analysis. BMJ Open 2018;8:e020464. doi:10.1136/ bmjopen-2017-020464

- Prepublication history for this paper is available online. To view these files, please visit the journal online (http://dx.doi. org/10.1136/bmjopen-2017020464).

Received 4 November 2017

Revised 6 April 2018

Accepted 18 April 2018

\section{ABSTRACT}

Introduction Continuous ambulatory peritoneal dialysis (CAPD) is the ideal modality for renal replacement therapy in most African settings given that it is relatively cheaper than haemodialysis (HD) and does not require in-centre care. CAPD is, however, not readily utilised as it is often complicated by peritonitis leading to high rates of technique failure. The objective of this study is to assess the prevalence of CAPD-related peritonitis and all-cause mortality in patients treated with CAPD in Africa.

Methods and analysis We will search PubMed, EMBASE, SCOPUS, Africa Journal Online and Google Scholar for studies conducted in Africa from 1 January 1980 to 30 June 2017 with no language restrictions. Eligible studies will include cross-sectional, prospective observational and cohort studies of patients treated with CAPD. Two authors will independently screen, select studies, extract data and conduct risk of bias assessment. Data consistently reported across studies will be pooled using randomeffects meta-analysis. Heterogeneity will be evaluated using Cochrane's $Q$ statistic and quantified using I $^{2}$ statistics. Graphical and formal statistical tests will be used to assess for publication bias.

Ethics and dissemination Ethical approval will not be needed for this study as data used will be extracted from already published studies. Results of this review will be published in a peer-reviewed journal and presented at conferences. The Preferred Reporting Items for Systematic reviews and Meta-Analyses for Protocols 2015 (PRISMA-P 2015) framework guided the development of this protocol. PROSPERO registration number CRD42017072966.

\section{INTRODUCTION}

Continuous ambulatory peritoneal dialysis (CAPD) ought to be the ideal modality for renal replacement therapy in most African settings, as it provides superior rehabilitation, better quality of life, averts need for expensive machines and allows for home-based therapy especially for patients in rural areas where immense distance from haemodialysis units

\section{Strengths and limitations of this study}

- To the best of our knowledge, there is no systematic review on the prevalence of continuous ambulatory peritoneal dialysis (CAPD)-related peritonitis in Africa, and this review will assist to fill the gap that exists in the literature.

- The findings of this review will aid policy and guidelines for the management of patients with CAPDrelated peritonitis.

- We will conduct a meta-analysis from the studies included.

- Limitation to this study is the low utilisation of CAPD in Africa and therefore few published studies are included in the analysis.

- Heterogeneity of studies carried out on the topic in Africa will be another limitation of this study.

exists. ${ }^{1}$ Africa is home to $70 \%$ of the least developed countries in the world and has a high burden of patients with chronic kidney disease $(\mathrm{CKD})$ with reported prevalence as high as $13.9 \% .{ }^{23}$ In 2007 , Africa's overall dialysis population accounted for $4.5 \%$ of the global dialysis population, with a total dialysis prevalence of 74 per million population (pmp), compared with a global average of 250 pmp. ${ }^{4}$ During the same period, the prevalence of CAPD in Africa was 2.2 pmp relative to global prevalence of $27 \mathrm{pmp}$, while $85 \%$ of all CAPD patients in Africa resided in South Africa. ${ }^{4}$ Challenges associated with utilisation of CAPD in Africa includes high cost of CAPD fluids (given that they are mostly imported), lack of expertise on utilisation of CAPD and several sociodemographic factors (including poor housing, overcrowding in houses, poor literacy, inadequate water and electricity 
supplies) that have been associated with peritonitis from various studies. ${ }^{135}$

Peritonitis remains the most common complication of CAPD in treated patients with end-stage renal disease (ESRD) ${ }^{6-8}$ Globally, CAPD-related peritonitis rates are estimated to be $0.24-1.66$ episodes per patient-year, ${ }^{9}$ exceeding, in many low-income and middle-income countries, the guidelines recommendation of not more than 0.5 episodes per patient-year. ${ }^{8}$ Although developed countries have observed a gradual decline in the prevalence of CAPD-related peritonitis due to improvements in the CAPD techniques, the same trends have not been reported in low-income and middle-income countries. ${ }^{10}$ Various sociodemographic factors, including availability of electricity and tap water, number of occupants at home,${ }^{5}$ level of education, distance from the dialysis units $^{11}$ as well as clinical and biochemical factors ${ }^{12}$ have been associated with CAPD-related peritonitis in Africans. ${ }^{512} 13$

CAPD-related peritonitis is independently associated with higher risk of all-cause, cardiovascular and infection-related mortality and this risk increases with prolonged use of CAPD. ${ }^{14}$ It is estimated that for every 0.5 per-year increase in peritonitis rate, the risk of death increases by $4 \%-18 \%{ }^{7}$ In one study, ${ }^{15}$ although the rate of peritonitis was found to be 0.60 episodes per patientyears, mortality among CAPD patients was reported to be $5.9 \%$ while peritonitis was directly implicated in $15.2 \%$ of all deaths, and non-peritonitis-related infections caused $68.5 \%$ of deaths. ${ }^{15}$ Another study from China showed a much higher mortality rate $(19.8 \%)$ despite reporting a low rate of peritonitis (0.16 episodes per patient-year). ${ }^{14}$ In the same study, peritonitis was independently associated with a $95 \%$ increase in all-cause mortality and up to fourfold risk of infection-related mortality. ${ }^{14}$ Abu-Aisha et al from Sudan reported a peritonitis rate of 0.55 episodes per patient-year with an overall mortality $10 \%$, of which $16.7 \%$ were related to peritonitis. ${ }^{16}$ Although these studies show that the prevalence of CAPD-related peritonitis is high and that overall mortality and peritonitis-related mortality is high in these patients, prevalence of peritonitis and mortality in African patients with ESRD treated with CAPD has not been adequately characterised. The purpose of this review is therefore to determine the prevalence of peritoneal dialysis-associated peritonitis as well as mortality reported in CAPD patients in Africa.

\section{OBJECTIVE}

To conduct a systematic review and meta-analysis to estimate the prevalence of peritonitis and mortality in African patients with ESRD treated with CAPD.

\section{REVIEW QUESTIONS}

This review aims to answer the following questions:

1. What is the prevalence of peritonitis among patients with ESRD treated with CAPD in Africa?
2. What is the mortality rate among patients treated with CAPD and in those who develop CAPD-related peritonitis?

3. What are the causative organisms for PD peritonitis?

4. What are the rates of modality switch to haemodialysis in patients with ESRD treated with CAPD who develop CAPD-related peritonitis?

\section{METHODS AND ANALYSIS}

The Preferred Reporting Items for Systematic reviews and Meta-Analyses for Protocols 2015 (PRISMA-P 2015) was

\begin{tabular}{ll}
\hline Table 1 & Search strategy for pubMed \\
\hline \multicolumn{3}{|c}{ Search terms } \\
\hline$\# 1$ & $\begin{array}{l}(((((\text { Peritoneal Dialysis) OR Continuous Ambulatory } \\
\text { Peritoneal Dialysis) OR Automated Peritoneal }\end{array}$ \\
Dialysis) OR CAPD) OR APD) \\
\#2 $\quad \begin{array}{l}((((\text { Peritonitis) OR Peritoneal infection) OR Peritoneal } \\
\text { Dialysis Peritonitis) OR PD Peritonitis) }\end{array}$
\end{tabular}

\#3 $\quad((()(()((\langle$ 'Africa”[MeSH] OR Africa*[tw] OR Algeria[tw] OR Angola[tw] OR Benin[tw] OR Botswana[tw] OR "Burkina Faso"[tw] OR Burundi[tw] OR Cameroon[tw] OR "Canary Islands" [tw] OR "Cape Verde"[tw] OR "Central African Republic"[tw] OR Chad[tw] OR Comoros[tw] OR Congo[tw] OR "Democratic Republic of Congo"[tw] OR Djibouti[tw] OR Egypt[tw] OR "Equatorial Guinea" [tw] OR Eritrea[tw] OR Ethiopia[tw] OR Gabon[tw] OR Gambia[tw] OR Ghana[tw] OR Guinea[tw] OR "Guinea Bissau"[tw] OR "Ivory Coast"[tw] OR "Cote d'Ivoire"[tw] OR Jamahiriya[tw] OR Jamahiryia[tw] OR Kenya[tw] OR Lesotho[tw] OR Liberia[tw] OR Libya[tw] OR Libia[tw] OR Madagascar[tw] OR Malawi[tw] OR Mali[tw] OR Mauritania[tw] OR Mauritius[tw] OR Mayote[tw] OR Morocco[tw] OR Mozambique[tw] OR Mocambique[tw] OR Namibia[tw] OR Niger[tw] OR Nigeria[tw] OR Principe[tw] OR Reunion[tw] OR Rwanda[tw] OR "Sao Tome"[tw] OR Senegal[tw] OR Seychelles[tw] OR "Sierra Leone"[tw] OR Somalia[tw] OR "South Africa"[(w] OR "St Helena"[tw] OR Sudan[tw] OR Swaziland[tw] OR Tanzania[tw] OR Togo[tw] OR Tunisia[tw] OR Uganda[tw] OR "Western Sahara"[tw] OR Zaire[tw] OR Zambia[tw] OR Zimbabwe[tw] OR "Central Africa" [tw] OR "Central African"]tw] OR "West Africa"[tw] OR "West African"[tw] OR "Western Africa"[tw] OR "Western African"[tw] OR "East Africa"[tw] OR "East African" [tw] OR "Eastern Africa"[tw] OR "Eastern African" [tw] OR "North Africa" [tw] OR "North African"[tw] OR "Northern Africa" [tw] OR "Northern African"[tw] OR "South African"[tw] OR "Southern Africa" [tw] OR "Southern African" [tw] OR "sub Saharan Africa" [tw] OR "sub Saharan African" [tw] OR "subSaharan Africa" [tw] OR "subSaharan African"[tw]) NOT ("guinea pig"[tw] OR "guinea pigs"(tw] OR "aspergillus niger"[tw] ))) )) )) )) ))))

\#4 \#1+\#2 + \#3

\#5 Limits \#4: Humans, 1980/01/01 to 2017/06/30 
used to develop the current protocol. ${ }^{17}$ PROSPERO registration number: CRD42017072966.

\section{Inclusion criteria}

All human studies meeting the criteria listed below will be included:

1. Observational studies (cross-sectional studies, prospective observational studies and cohort studies) and letters to editors reporting on the prevalence of peritonitis and all-cause mortality among children and adults with ESRD treated with CAPD and residing in Africa.

2. Age: there will be no age restriction.

3. Duration: we will consider data from all studies published from the 1 January 1980 to 30 June 2017.

4. Language restrictions: there will be no language restriction.

5. Sample size restrictions: there will be no sample size restriction.

\section{Exclusion criteria}

The following types of studies will not be considered:

1. Case reports, case-control studies, randomised control trials and review articles.
2. Studies conducted among populations of African origin residing outside of Africa.

3. Duplicates: for studies published in more than one paper, the most comprehensive one reporting the largest sample size will be considered.

4. Studies from which the prevalence of PD-related peritonitis cannot be determined and whose full data could not be accessed even after request from the authors.

\section{Source of information}

This systematic review will be reported according to the Meta-Analysis of Observational Studies in Epidemiology Guidelines. ${ }^{18}$

\section{Search strategy for identifying related studies}

The search strategy will be conducted in two stages.

Bibliographic database searches

Relevant studies will be identified by searching PubMed, EMBASE, SCOPUS, Africa Journal Online and Google Scholar. The searches will only be limited to studies performed in Africa on African subjects and published from 1 January 1980 to 30 June 2017. Key search terms will be: 'peritoneal dialysis', 'peritonitis', 'Africa' with a filter

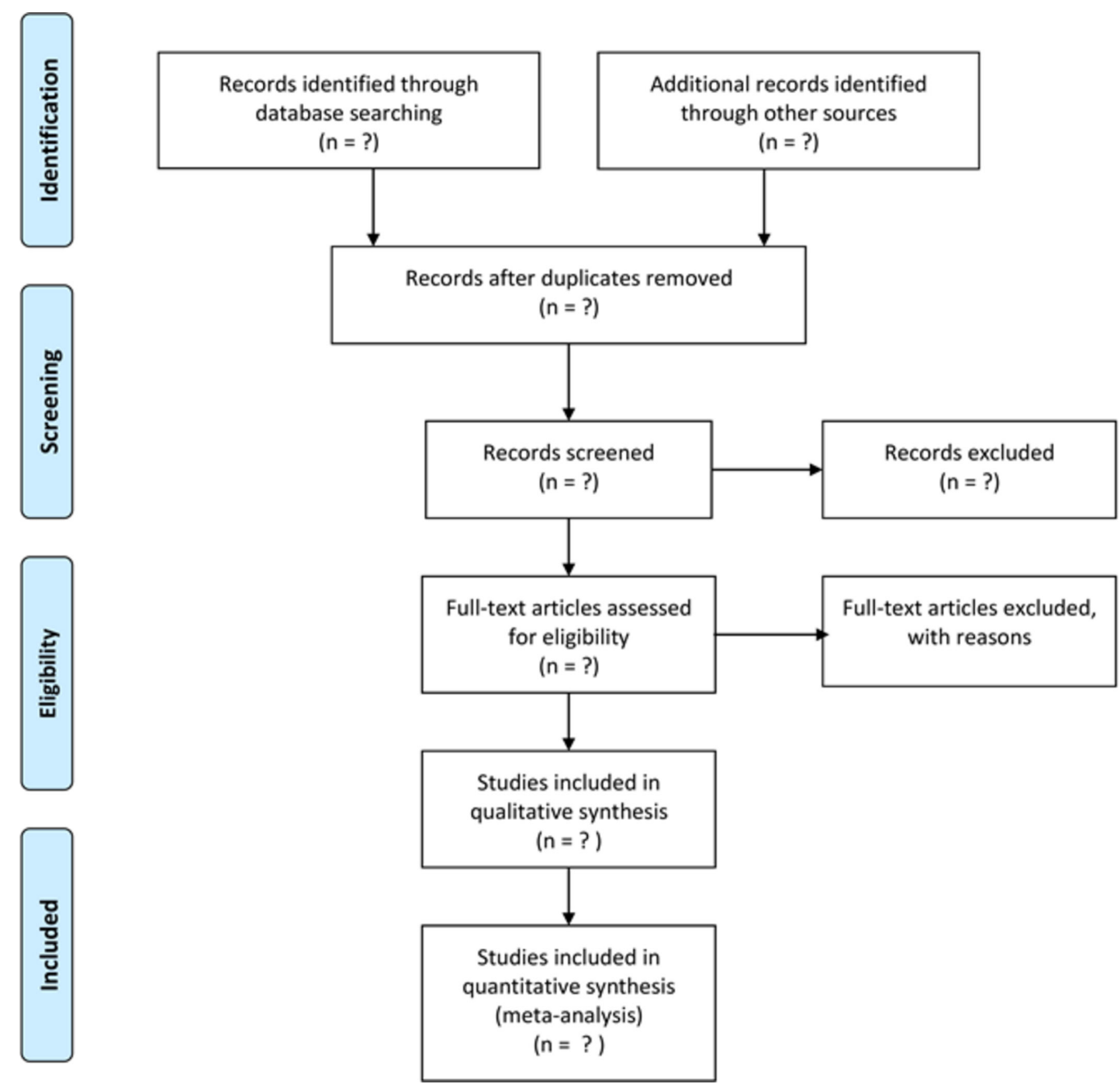

Figure 1 Flow diagram for study selection. 
to include all African countries (PubMed search strategy depicted on table 1). We will further use controlled vocabularies synonyms to identify related terms. Individual country names for all African countries will also be included in the search. Two authors (MWM and SK) will independently conduct the database searches and articles in French will be assessed by (JJN).

\section{Searching for other sources}

We will further manually scan through the reference lists of pertinent studies in search of more studies. Authors for studies which cannot be accessed through online searches will be directly contacted via email to request for the articles. Where we are unable to get feedback from the author, the studies will be excluded from the review.

\section{Selection of studies for inclusion in the review}

An abstract of articles reporting on the prevalence and outcomes of CAPD peritonitis will be screened for inclusion into the review. Titles and abstracts of all eligible papers will be independently reviewed by two authors (MWM and SK). A joint review process will be undertaken for any disagreement during the selection process and one of the authors (IGO) will be asked to adjudicate the process in order to reach consensus. Figure 1 shows the process of study selection for the review.

\section{Assessment of methodological quality and reporting of data}

Two authors (MWM and SK) will assess the quality of eligible studies guided by the Joanna Briggs Institute Critical Appraisal Checklist for Studies Reporting Prevalence Data (2014) and the guidelines from the Cochrane Handbook for Systematic Reviews of Interventions V. 5.1.0. ${ }^{19} 20$

\section{Patients and public involvement}

Patients and public were not involved in the marking of this study protocol.

\section{Data extraction and management}

Two investigators (MWM and SK) will independently extract data from included studies, using a standardised and pretested data extraction form. Extracted data will include study characteristics (authors, country, year of publication, the study design, sample size, mean or median age of population, gender distribution, duration of study and mean or median duration of CAPD use) and specific features related to complications of CAPD (number of episodes of peritonitis and rates, causative organisms of peritonitis and rates of technique failure or modality switch to HD). Data on overall mortality and mortality related to peritonitis will also be extracted from each study.

\section{Data synthesis and analysis}

All data will be analysed using the $\mathrm{R}$ statistical software (V.3.3.3 (2017-03-06), the R Foundation for statistical computing, Vienna, Austria) and 'meta' package. Interrater agreement for study inclusion will be assessed using Cohen's $\kappa$ coefficient. ${ }^{21}$ For data that we are unable to conduct a meta-analysis, we will provide a narrative description. These data will include study characteristics such as year of publication, sample size and country where study. Peritonitis rates will be reported as number of episodes per patient-year. ${ }^{8}$ For the outcomes of interest consistently reported across studies, random effects model meta-analyses will be used to pool estimates across those studies. Stratified analyses will be consistently used to derive those pooled estimate separately in adult and paediatric populations. Heterogeneity across studies will be evaluated using Cochrane's $Q$ statistic and quantified using $\mathrm{I}^{2}$ statistics. Where substantial heterogeneity is detected, a subgroup analysis will be performed to explore the possible sources using the following grouping variables: age group, gender, geographical area (central, eastern, northern, southern and western Africa) and study quality. Graphical and formal statistical tests will be used to assess for publication bias. ${ }^{20}$ Potential outliers will be investigated in sensitivity analysis by dropping each study at a time. The Duval and Tweedie trim-and-fill will be used to adjust estimates for the effects of publication bias.

\section{Presentation and reporting of results}

The PRISMA flow chart will be used to show how the studies were included into the review and also explain why some of the studies were excluded. Data will be summarised in tables and forest plots. Where appropriate, pie charts will also be used.

\section{ETHICS AND DISSEMINATION}

Ethical approval will not be needed for this study as data used will be extracted from already published studies. Results of this review will be published in a peer-reviewed journal and presented at conferences.

\section{Author affiliations}

${ }^{1}$ Division of Nephrology and Hypertension, University of Cape Town, Cape Town, South Africa

${ }^{2}$ Department of Medicine, Princess Marina Hospital, Gaborone, Botswana ${ }^{3}$ Kidney and Hypertension Research Unit, University of Cape Town, Cape Town, South Africa

${ }^{4}$ Division of Clinical Practice and Patient Care, National University of Science and Technology, Bulawayo, Zimbabwe

${ }^{5}$ Department of Medicine, Groote Schuur Hospital, University of Cape Town, Cape Town, South Africa

${ }^{6}$ Department of Medicine, Federal Medical Centre, Keffi, Nigeria

${ }^{7}$ Department of Medicine, University of Uyo, Uyo, Nigeria

${ }^{8}$ Non-communicable Diseases Research Unit, South African Medical Research Council, Cape Town, South Africa

${ }^{9}$ Division of Nephrology and Immunology, Department of Medicine, University of Alberta, Edmonton, Alberta, Canada

Contributors MWM and SK designed the protocol and JJN translated papers written in French. MWM drafted the manuscript. SK, JJN, IOM, AKB and IGO revised the manuscript for intellectual content while UE and APK performed meta-analysis of the data. All authors approved the final version of the manuscript.

Funding The authors have not declared a specific grant for this research from any funding agency in the public, commercial or not-for-profit sectors.

Competing interests None declared. 
Patient consent Not required

Provenance and peer review Not commissioned; externally peer reviewed.

Data sharing statement Not applicable.

Open Access This is an Open Access article distributed in accordance with the Creative Commons Attribution Non Commercial (CC BY-NC 4.0) license, which permits others to distribute, remix, adapt, build upon this work non-commercially, and license their derivative works on different terms, provided the original work is properly cited and the use is non-commercial. See: http://creativecommons.org/ licenses/by-nc/4.0/

(C) Article author(s) (or their employer(s) unless otherwise stated in the text of the article) 2018. All rights reserved. No commercial use is permitted unless otherwise expressly granted.

\section{REFERENCES}

1. Nayak KS, Prabhu MV, Sinoj KA, et al. Peritoneal dialysis in developing countries. Contrib Nephrol 2009;163:270-7.

2. Stanifer JW, Jing B, Tolan S, et al. The epidemiology of chronic kidney disease in sub-Saharan Africa: a systematic review and metaanalysis. Lancet Glob Health 2014;2:e174-81.

3. Okpechi IG, Rayner BL, Swanepoel CR. Peritoneal dialysis in Cape Town, South Africa. Perit Dial Int 2012;32:254-60.

4. Abu-Aisha H, Elamin S. Peritoneal dialysis in Africa. Perit Dial Int 2010;30:23-8.

5. Zent R, Myers JE, Donald D, et al. Continuous ambulatory peritoneal dialysis: an option in the developing world? Perit Dial Int 1994;14:48-51.

6. Katz IJ, Sofianou L, Hopley M. An African community-based chronic ambulatory peritoneal dialysis programme. Nephrol Dial Transplant 2001;16:2395-400.

7. Nieto-Ríos JF, Díaz-Betancur JS, Arbeláez-Gómez M, et al. Peritoneal dialysis-related peritonitis: twenty-seven years of experience in a Colombian medical center. Nefrologia 2014;34:88-95.

8. Li PK, Szeto CC, Piraino B, et al. ISPD peritonitis recommendations: 2016 update on prevention and treatment. Peritoneal Dial Int 2016;36:481-508.
9. Akoh JA. Peritoneal dialysis associated infections: An update on diagnosis and management. World J Nephrol 2012;1:106.

10. van Esch S, Krediet RT, Struijk DG. 32 years' experience of peritoneal dialysis-related peritonitis in a university hospital. Perit Dial Int 2014;34:162-70.

11. Isla RA, Mapiye D, Swanepoel CR, et al. Continuous ambulatory peritoneal dialysis in Limpopo province, South Africa: predictors of patient and technique survival. Perit Dial Int 2014;34:518-25.

12. Raaijmakers R, Gajjar P, Schröder C, et al. Peritonitis in children on peritoneal dialysis in Cape Town, South Africa: epidemiology and risks. Pediatr Nephrol 2010;25:2149-57.

13. Swanepoel CR, Wearne N, Okpechi IG. Nephrology in Africa - not yet uhuru. Nat Rev Nephro/2013;9:610-22.

14. Ye H, Zhou Q, Fan L, et al. The impact of peritoneal dialysisrelated peritonitis on mortality in peritoneal dialysis patients. BMC Nephrol2017;18:186.

15. Pérez Fontan M, Rodríguez-Carmona A, García-Naveiro R, et al. Peritonitis-related mortality in patients undergoing chronic peritoneal dialysis. Perit Dial Int 2005;25:274-84.

16. Abu-Aisha $\mathrm{H}$, Elhassan $\mathrm{EA}$, Khamis $\mathrm{AH}$, et al. Rates and causes of peritonitis in a National Multicenter Continuous Ambulatory Peritoneal Dialysis program in Sudan: first-year experience. Saudi J Kidney Dis Transpl 2007;18:565-70.

17. Moher D, Shamseer L, Clarke M, et al. Preferred reporting items for systematic review and meta-analysis protocols (PRISMA-P) 2015 statement. Syst Rev 2015;4:1.

18. Stroup DF, Berlin JA, Morton SC, et al. Meta-analysis of observational studies in epidemiology: a proposal for reporting. Meta-analysis Of Observational Studies in Epidemiology (MOOSE) group. JAMA 2000;283:2008-12.

19. The Joanna Briggs Institute Levels of Evidence and Grades of Recommendation Working Party. Supporting Document for the Joanna Briggs Institute Levels of Evidence and Grades of Recommendation [Internet]. Joanna Briggs Inst 2014:1-18 http:// joannabriggs.org/assets/docs/approach/Levels-of-EvidenceSupportingDocuments-v2.pdf.

20. Higgins JP, Green S. Cochrane handbook for systematic reviews of interventions version 5.1.0 [Internet]. London: The Cochrane Collaboration, 2011.

21. Sedgwick P. Cohen's coefficient к. BMJ 2012;344:e1178. 